\title{
Safety of coprescribing NSAIDs with multiple antihypertensive agents
}

Triple drug combinations are associated with increased hospital admission for acute kidney injury, but questions remain

\author{
Dorothea Nitsch clinical senior lecturer, Laurie A Tomlinson honorary research fellow
}

Department of Non-Communicable Disease Epidemiology, Faculty of Epidemiology and Population Health, London School of Hygiene and Tropical Medicine, London WC1E 7HT, UK

Current guidelines by the National Institute for Health and Clinical Excellence (NICE) recommend treatment with angiotensin converting enzyme (ACE) inhibitors and angiotensin receptor blockers (ARBs) for conditions such as hypertension, ${ }^{1}$ chronic heart failure, ${ }^{2}$ and proteinuric chronic kidney disease. ${ }^{3}$ In England, the prescription of these drugs has increased by $15.8 \%$ over the past four years. ${ }^{4}$ Because ACE inhibitors and ARBs are often coprescribed with non-steroidal anti-inflammatory drugs (NSAIDs) and diuretics, particularly in older people, ${ }^{5}$ we need to know more about the safety of such combinations.

In a linked paper (doi:10.1136/bmj.e8525), Lapi and colleagues used a large database that was representative of UK primary care to examine data on 487372 patients taking antihypertensive drugs. ${ }^{6}$ They used a nested case-control design to examine whether adding an NSAID to an ACE inhibitor, ARB, or diuretic in double or triple therapy combinations increased the risk of subsequent hospital admission with acute kidney injury. Acute kidney injury is seen in more than $20 \%$ of hospital inpatients and is associated with around half of all potentially preventable deaths in hospital. ${ }^{78}$ Although recent observational evidence suggests a link between this condition and use of diuretics, NSAIDs, ACE inhibitors, and ARBs, ${ }^{9}$ such studies are confounded by indication. Patients who are prescribed a combination of these agents are usually at high risk of acute kidney injury, which makes it difficult to establish a causal association.

The authors of the current study used a strict case definition of acute kidney injury: cases were defined using the first diagnostic code and those in which acute kidney injury was just a secondary problem were excluded. They excluded people with chronic kidney disease and adjusted for other comorbidities in the analysis. Chronic kidney disease is associated with an increased risk of acute kidney injury and well informed patients may avoid taking NSAIDs, which could introduce confounding by contraindication. ${ }^{10}$
Adjusted analyses found a $31 \%$ higher risk of acute kidney injury (relative risk $1.31,95 \%$ confidence interval 1.12 to 1.53 ) for a triple drug combination (adding an NSAID to an ACE inhibitor or ARB plus diuretic) but no clear evidence of an increased risk of acute kidney injury for double drug combinations (NSAID added to either a diuretic, ACE inhibitor, or ARB). These results remained consistent after several sensitivity analyses.

So can clinicians be confident that NSAIDs in double combination with an ACE inhibitor, ARB, or diuretic are not associated with acute kidney injury? Defining safety as the absence of adverse events provides us with the difficult statistical challenge of excluding the presence of associations. To make sure that a particular drug combination is safe, an extremely large dataset of a representative patient population must be randomised to a given drug combination versus a single drug class, with frequent follow-up to detect adverse events.

In the current study, confidence intervals for estimates of risk for double drug combinations were wide, so the evidence of safety is not strong. Indeed, there was a suggestion of an early increase in risk for a diuretic-NSAID combination. Secondly, this analysis could not adjust for over-the-counter NSAID use or tell us about instances when primary care doctors detected increases in serum creatinine and stopped drugs before patients needed hospital admission. Thirdly, drug associated acute kidney injury is often a complication of other illnesses. ${ }^{11}$ Lastly, defining acute kidney injury in patients with chronic kidney disease is complex, but it is important to understand how acute kidney injury can be prevented in this high risk group. ${ }^{10}$ Therefore, Lapi and colleagues' study probably underestimates the true burden of drug associated acute kidney injury. The jury is still out on whether double drug combinations are indeed safe.

The implications of the current analysis are nevertheless important: clinicians must advise patients who are prescribed diuretics, ACE inhibitors, or ARBs of the risks associated with NSAID use and they must also be vigilant for signs of drug associated acute kidney injury in all patients. Importantly, 
current NICE quality standards for people with chronic kidney disease advise a drug review and renal function check during acute illness. $^{12}$

Lapi and colleagues' paper highlights how observational data can improve our understanding of the risk to benefit ratio of drugs in routine use in the general population (as opposed to the defined populations where initial clinical trials were undertaken). Work still needs to be done to understand whether drug associated acute kidney injury is preventable, and what role intercurrent illness plays. The role of prescribed drugs in increasing the severity or duration of hospital admission may be as important as single organ complications in the elderly population with multiple comorbidities to whom these drugs are mainly prescribed. The current study is an important step in the right direction, but a longer road of discovery is ahead.

Competing interests: Both authors have completed the ICMJE uniform disclosure form at www.icmje.org/coi_disclosure.pdf (available on request from the corresponding author) and declare: no support from any organisation for the submitted work; no financial relationships with any organisations that might have an interest in the submitted work in the previous three years; no other relationships or activities that could appear to have influenced the submitted work.

Provenance and peer review: Commissioned; not externally peer reviewed.

1 National Institute for Health and Clinical Excellence. Hypertension: clinical management of primary hypertension in adults. CG127. 2011. http://publications.nice.org.uk/ hypertension-cg127.
2 National Institute for Health and Clinical Excellence. Chronic heart failure: management of chronic heart failure in adults in primary and secondary care. CG108. 2010. http:// publications.nice.org.uk/chronic-heart-failure-cg108.

3 National Institute for Health and Clinical Excellence. Chronic kidney disease: early identification and management of chronic kidney disease in adults in primary and secondary care. CG78. 2008. http://publications.nice.org.uk/chronic-kidney-disease-cg73.

4 Tomlinson LA, Payne RA, Abel GA, Chaudhry AN, Tomson CV, Wilkinson IB, et al. Relation between national changes in prescription of angiotensin-converting enzyme inhibitors and angiotensin receptor blockers and admissions with acute kidney injury. Lancet 2012. Abstract 72. www.thelancet.com/abstracts/public-health-science-in-the-uk.

5 Loboz KK, Shenfield GM. Drug combinations and impaired renal function-the "triple whammy." Br J Clin Pharmacol 2005;59:239-43.

6 Lapi F, Azoulay L, Yin H, Nessim SJ, Suissa S. Concurrent use of diuretics, angiotensin converting enzyme inhibitors, and angiotensin receptor blockers with non-steroidal anti-inflammatory drugs and risk of acute kidney injury: nested case-control study. BMJ 2013;346:e8525.

7 Stewart JFG, Smith N, Kelly K, Mason M. Adding insult to injury: a review of the care of patients who died in hospital with a primary diagnosis of acute kidney injury (acute renal failure). A report by the National Confidential Enquiry into Patient Outcome and Death. 2009. www.ncepod.org.uk/2009report1/Downloads/AKI report.pdf.

8 Wang HE, Muntner P, Chertow GM, Warnock DG. Acute kidney injury and mortality in hospitalized patients. Am J Nephrol 2012;35:349-55.

9 Fournier JP, Sommet A, Durrieu G, Poutrain JC, Lapeyre-Mestre M, Montastruc JL. Drug interactions between antihypertensive drugs and non-steroidal anti-inflammatory agents: a descriptive study using the French Pharmacovigilance database. Fundam Clin Pharmacol 2012; published online 2 Nov.

10 Hsu CY, Ordonez JD, Chertow GM, Fan D, McCulloch CE, Go AS. The risk of acute renal failure in patients with chronic kidney disease. Kidney Int 2008;74:101-7.

11 Plataki M, Kashani K, Cabello-Garza J, Maldonado F, Kashyap R, Kor DJ, et al. Predictors of acute kidney injury in septic shock patients: an observational cohort study. Clin J Am Soc Nephrol 2011;6:1744-51.

12 National Institute for Health and Clinical Excellence. Chronic kidney disease quality standard. 2011. http://publications.nice.org.uk/chronic-kidney-disease-quality-standardqs5.

Cite this as: BMJ 2013;346:e8713

(c) BMJ Publishing Group Ltd 2013 